\title{
II. On a wedge-and-diaphragm photometer
}

\section{Robert Sabine}

To cite this article: Robert Sabine (1883) II. On a wedge-and-diaphragm photometer , Philosophical Magazine Series 5, 15:91, 22-28, DOI: 10.1080/14786448308627301

To link to this article: http://dx.doi.org/10.1080/14786448308627301

曲 Published online: 28 Apr 2009.

Submit your article to this journal $\pi$

Џll Article views: 2

Q View related articles $\asymp$ 
already been led by my examination of the observations of other physicists on the passage of electricity through rarefied gases. The maximum attained by the current-intensity at a certain pressure of the air when a current traverses a space of rarefied air is not due in any way, as it has been generally assumed to be, to the resistance $r_{1} l$ of the air having its minimum at that pressure, and afterwards increasing in amount with the increase of rarefaction, but really to this-that the sum $e+r_{1} l$ then possesses its minimum value. With the continuation of the rarefaction the resistance $r_{1} l$ continues to diminish, while $e$ incessantly increases. Consequently the circumstance mentioned above, namely that the induced current possesses its maximum value at a certain pressure of air, gives no support to the allegation that in highly rarefied air or in a vacuum the resistance is sufficiently great to prevent the current passing. Here it is not the resistance of the gas, but the electromotive force $e_{1}$ increasing with the rarefaction and connected with the electrodes, that presents an obstacle to the passage of the current. Every thing is in favour of the hypothesis that vacuum opposes a very feeble resistance to the propagation of electricity. One can therefore, without the employment of electrodes, by induction at a distance, or by friction at the surface of a tube in which the air is sufficiently rarefied to render the passage of a strong induction-current between the electrodes impossible, easily excite in that tube an electric motion sufficiently considerable to produce a sensible development of light. Now this would be impossible if highly rarefied gas or a vacuum were an insulator.

\section{On a Wedge-and-Diaphragm Photometer. By Robert Sabine*.}

TTHE photometer described by me in a paper contributed to 1 the last Meeting of the British Association at Southamptont was based upon the weakening of the light to a constant value by the interposition of sheets of some absorbing material as far as could be, and finally obtaining a balance by varying the distance of the photometer from the light to be measured.

I find it, however, frequently in practice more convenient to keep the photometer at a constant distance from the source of light, and to effect a balance by the gradual increase of the thickness of the absorbing material only, using at the same

* Communicated by the Author.

$\dagger$ Electrical Review, vol. xi. p. 197. 
time different illuminations of the field of comparison for extending the range of the instrument.

The photometer I have constructed is shown in perspective in the figure. It consists of a brass tube mounted upon a

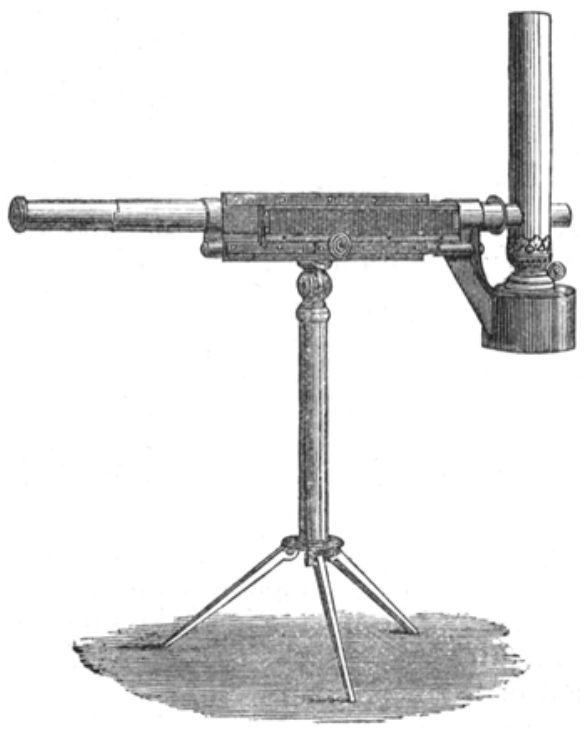

stand, and having an eyepiece at one end and a paraffin-lamp at the other. The light of the paraffin-flame, entering at the end of the tube, falls upon a disk of opal glass placed near the middle of the tube, and keeps it constantly illuminated. In the middle, the tube is cut away and a collar fitted over it, which can be turned into any required position. This collar has at one side of it a slit containing a strip of opal glass, opposite which it has fixed to it a frame carrying a wedge of neutral-tinted shade glass, corrected by being combined with a reversed wodge of white glass, with which it is mounted in a slide with rack-and-pinion adjustment. The thicker end of the wedge absorbs about eight times as much light as the thinner end; that is to say, the light from eight candles, after passing through the thicker end, has the same illuminating effect as the light of one candle after passing through the thinner end.

When the light to be measured, which is placed on the right-hand side of the photometer, is above or below the horizontal, the wedge is turned, with the collar which carries it, so that the rays from the light, whatever may be its position, fall 
normally upon the face of the wedge. The light then passes through and is weakened by the wedge, then through the strip of opal glass, through the slit behind it into the interior. Here it is incident upon a small mirror made of a strip of silvered microscope covering-glass, placed at an angle of $45^{\circ}$ to the axis of the tube, and is thence reflected to the eye of the observer. The other end of the photometer, close to the bright part of the paraffin-flame, is furnished with a cap containing a circular diaphragm disk with three different apertures, either of which can be inserted by simply turning the disk. The little mirror is supported from the face of the constantly illuminated opal pane in such a way that the support is hidden from the observer by the mirror. The object of this arrangement is to ensure the observed surfaces whose illuminations are to be compared being in juxtaposition without any apparent division-line or separation. It is well known* that when the two surfaces to be compared are at a perceptible distance apart, as in Rumford's photometer, no certainty of accurate measurement is possible. The method of supporting a small mirror in the centre of the field of comparison in the way described is very satisfactory. The glass of the mirror being extremely thin, I find with a moderate illumination, and when the light reflected by the mirror is of the same colour as that of the field, the mirror sometimes entirely vanishes from sight at the moment of balance.

The distance at which the light to be observed is placed from the photometer varies, of course, according to convenience; but where it is optional, I prefer for all lights under 1000 candles a constant distance of 1 metre. Stronger lights become inconvenient to the observer if placed so near; and in that case $I$ increase the distance accordingly, while of course at the same time increasing the range of the photometer.

The degree of illumination of the field of comparison is adjustable by means of the diaphragms. The smallest diaphragm illuminates the field so that the light of a single candle can be conveniently balanced. The next diaphragm exposes an area about eight times as large of the bright portion of the paraffin-flame, and the largest diaphragm about sixty-four times; that is to say, it has about eight times the diameter of the smallest diaphragm. The resulting degrees of illumination of the field can be thus calculated as well as compared by actual observation. Let the illumination of the field with these three diaphragms be respectively $\lambda_{1}, \lambda_{2}, \lambda_{3}$. Of the light which falls upon the outer face of the wedge a

* Herschel, 'On Light,' p. 29; Helmholtz, Physiologische Optik, p. 329. 
fraction is, of course, reflected and lost; the remaining fraction $(s)$ enters the wedge. Let $l$ be the illuminating-power of the light (say a standard candle) at a unit distance from it, in the direction of the photometer, $n$ the thickness of the wedge at the point interposed, $m$ its coefficient of transmission, and $d$ the distance of the light from the opal pane in the slit; then the illuminating-power of the light which reaches the opal pane will be

$$
\frac{s \ln n}{d^{2}}
$$

This light falls upon the surface of the opal glass; a fraction enters it, part of which is absorbed, the remainder passing to the mirror, a further part boing absorbed in the reflection to the eye of the observer. After the further diminution up to this point the fraction of the light which leaves the opal pane and which actually reaches the eye is $s_{1}$, the apparent illumination of the mirror with the smallest diaphragm being

$$
\lambda_{1}=\frac{s s_{1} l m r^{n}}{d^{2}} \text {. }
$$

Similarly, if we now remove the candle and substitute some other light whose illuminating-power at unit distance is $\mathrm{L}$ and its actual distance $\mathrm{D}$, the wedge will have to be readjusted to $n_{1}$; and if beyond the range of the wedge, the field will have to be adjusted by the diaphragm being increased (say to the illumination of the field $\lambda_{2}$ ); then

$$
\lambda_{2}=\frac{s s_{1} \mathrm{~L} m^{n_{1}}}{\mathrm{D}^{2}} ;
$$

the relation of the illuminating-powers of the two lights will be

$$
\frac{\mathrm{L}}{l}=\left(\frac{\mathrm{D}}{d}\right)^{2} \frac{\lambda_{2}}{\lambda_{1}} m^{n-n_{1}}
$$

The value of $\frac{\lambda_{2}}{\lambda_{1}}$ is ascertained experimentally by obtaining a balance with some constant light at different distances, as well as by measuring the openings of the diaphragms. The values $d$ and $m^{n}$ for the standard candle are constants.

$$
\text { Let } \frac{m^{n}}{d^{2}}=\alpha \text {, then } \quad \frac{L}{l}=\frac{\lambda_{2} \alpha}{\lambda_{1} m^{n_{1}}} \mathrm{D}^{2} \text {. }
$$

The wedge is provided with a scale divided into millimetres, by which the position is observed. A table is constructed 
giving the values of $\frac{\alpha}{m^{n_{1}}}$, which is determined for each position of the wedge by obtaining a balance with a constant light at different distances, and for each of the diaphragms controlling the illumination of the comparison-field. In using the photometer in practice, it is therefore only necessary to obtain a balance and to read off the indication of the scale. A reference to the table then gives the value which, multiplied into the square of the distance in metres, gives the candle-power of the measured light, or, if the distance be exactly 1 metre, gives the candle-power direct.

It happens sometimes that the degree of illumination to bo ascertained is independent of distance, as in the case of daylight; in this case the diaphragm and wedge are adjusted until a balance is obtained. The value given by the table then represents the number of standard candles which at 1 metre distance would illuminate an object to an equal degree.

In measuring the illuminating-powers of lights the colours of the lights have sometimes to be taken into account. The way I prefer to do this is to assume the ingredient of orange light contained in daylight as unit for comparing the relative ingredient of orange light contained in the artificial light which is measured. By orange light I mean that ingredient of light which passes freely through orange glass, and which in fact embraces nearly all that portion of the visible spectrum between the lines B and F-that is to say, the red, orange, yellow, and a small part of the green rays; whilst with a moderate illumination, such as is used in photometric comparisons, all the more refrangible and less luminous rays from the line $\mathbf{F}$ upwards are quenched.

For convenience of observing, the photometer is furnished with a small disk centred inside the eyepiece and containing small panes of white and orange glass*, either of which can be instantly interposed when required.

A balance of illuminating effect is first estimated with the white pane irrespective of colour; then the disk is turned so as to interpose the orange pane, and the wedge readjusted accordingly. If with the orange glass the reading is $\left\{\begin{array}{l}\text { higher } \\ \text { lower }\end{array}\right\}$ than with the white (that is, if $\left\{\begin{array}{c}\text { less } \\ \text { more }\end{array}\right\}$ light has to be admitted through the wedge in order to maintain the balance),

* The disk contains also red and green glasses, and may be furnished with any other colours for which the observer has a fancy. 
it follows that the observed light contains proportionally $\left\{\begin{array}{c}\text { more } \\ \text { less }\end{array}\right\}$ orange than the paraffin-flame.

Thus with the white-glass pane daylight in the laboratory was balanced by a reading which the table indicated to be equal to 55 candles at 1 metre. Observed through the orange glass, however, the reading indicated the orange ingredients in the daylight to be equal to 42 candles; that is to say, with the paraffin-flame the light which passed through orange glass was $\frac{55}{42}=1 \cdot 3$ times the corresponding ingredient of the same coloured light in daylight. The light of an incandescent lamp through white glass was 28 candles, while through the orange glass it was 26 candles; therefore the incandescent lamp had $\frac{26}{28}=0.94$ time the corresponding colour in the paraffin-flame; and as the paraffin-flame had 1.3 as much as daylight, the incandescent lamp had $1.3 \times 0.94=1 \cdot 2$ as much as daylight. In other words, if we had a similar quantity of light obtained from daylight and from incandescent lamps, we should have in the latter about 20 per cent. more orange-tinted light.

This colour-comparison is of course only very rough; but it enables the excess of orange light in incandescent lamps, and its occasional deficiency in some arc lamps, to be approximately compared.

I have been led to employ in practice a selected portion of a paraffin-flame in preference to a standard candle, partly in consequence of the fact that the paraffin-flame can be enclosed in a light-tight lantern, but chiefly on account of its superior steadiness and constancy. As an instance, I have obtained a balance between selected portions of two paraffin-flames, which balance has been maintained for hours at a time without the slightest variation. On the other hand, when a standard candle was compared with the bright part of a paraffin-flame, the photometer had to be readjusted continually, showing several-per-cent. variations in the burning of the candle. I therefore prefer to take a long series of readings once for all in determining the value of the light admitted to the comparison-field of the photometer by each diaphragm, to assume means of these as constants of reduction to the nominal standard, and to depend upon the constancy and steadiness of the lights given through the diaphragms by the paraffin-flame afterwards. After working practically with this system for some time, iny opinion is that a selected area of the bright 
part of a paraffin-flame would be a much better unit of light than the present standard candle, particularly as distances would be measured from the diaphragm and not from the flame, by which error due to the curvature of the flame would disappear.

Grosmont House, Hampton Wick.

III. On Lockyer's Theory of Dissociation. By Hermann W. Voger.*

TN February 1880 I took an opportunity to criticise, on the ground of my observations of the spectrum of chemically pure hydrogen, Lockyer's view that calcium is dissociated at a very high temperaturef. Lockyer takes for his startingpoint, inter alia, that in the spectra of the so-called white stars, photographed by Huggins, only the first of the two calcium-lines $H^{\prime}$ and $\dot{H}^{\prime \prime}$ is present, and accordingly advances the theory that calcium at a high temperature separates into two substances $X$ and $Y$, of which the first gives the line $\mathrm{H}^{\prime}$, the other the line $\mathrm{H}^{\prime \prime}$, and that, in the stars alluded to, only the first exists. I showed, on the contrary, that hydrogen possesses, besides the four well-known readily seen lines, another, singular line with extremely intense photographic action, which nearly coincides with Fraunhofer's line $\mathbf{H}^{\prime}$, and that we are so much the more entitled to hold that the supposed calcium-line observed by Huggins is the fifth hydrogenline, as the known lines of hydrogen are especially well developed in the spectra of those stars, and also the ultraviolet star-lines observed by Huggins coincide with the ultraviolet hydrogen-lines photographically fixed by mef.

Lockyer, however, has not given up his notion about dissociation, but has sought in spectroscopy for now evidence in favour of it.

He remarks that, inter alia, in the spectrum of the sunspots certain iron-lines appear widened, others not, and, further, that many of them, as $\lambda 4918$ and $\lambda .4919 \cdot 7$, do not occur in the spectrum of the protuberances (which show other lines of iron), but certainly do in the spectrum of the spots, while in these, again, iron-lines which the former contain are, under some circumstances, wanting; and he then continues,

- Translated from the Sitzungsberichte der Königlich Preussischen Akademie der Wissenschaften zu Berlin, Nor. 2, 1882, pp. 905-907.

+ Proc. Roy. Soc. Ixviii. p. 157.

$\ddagger$ Monatsb. d. Berliner Akad. d. Wissenseh. 1880, p. 192. 